\title{
Potential and Carrier Distribution in AlGaN Superlattice
}

\author{
K.P. Korona*, K. Pakula, R. Bożek, A. Drabińska, \\ K. Surowiecka, R. StȩPniewski, E. Zielińska-Rohozińska
}

AND J.M. BARANOWSKI

Institute of Experimental Physics, Warsaw University

Hoża 69, 00-681 Warsaw, Poland

\begin{abstract}
Photocurrent spectroscopy and Kelvin force microscopy have been used in order to determine charge, field, and potential distributions in spontaneously grown superlattice. The spectra show that light can generate currents and potentials in both directions depending on photon energy. A numerical model made for superlattice of period $\lambda_{\mathrm{SL}}=33 \mathrm{~nm}$ shows that electric field in superlattice oscillates coherently with $\mathrm{Al}$ content. The oscillations of electric field explain the different directions of photocurrent. The electric field can also separate electrons and holes, making carrier lifetimes longer and lowering excitation intensity threshold for occupation inversion.
\end{abstract}

PACS numbers: 72.40.+w, 73.61.Ey, 73.21.Cd

\section{Introduction}

GaN/AlGaN structures exhibits many interesting properties that are related to internal electric fields caused by spontaneous and piezoelectric polarization. These polarizations result from differences of $\mathrm{Al}$ content in neighboring layers [1]. The field can cause electron-hole separation in quantum wells and dots leading to a longer carrier lifetime. Moreover, the field can be used to attract electrons which leads to the formation of two-dimensional electron gas [2] and can be used for polarization doping - an exciting technique for creating electron slabs with widely tunable density and confinement [3].

The strong polarization of AlGaN crystal can lead to the growth of structures with modulated $\mathrm{Al}$ content $X_{\mathrm{Al}}$ - spontaneously grown superlattice (SL) [4].

*corresponding author; e-mail: kkorona@fuw.edu.pl 
Here, we report on study of superlattice created by spontaneous oscillations of aluminum content $X_{\mathrm{Al}}$. We present measurements and numerical modeling of optical effects caused by electric field and potential oscillations in samples containing $\mathrm{Al}_{x} \mathrm{Ga}_{1-x} \mathrm{~N}$ with periodically modulated $X_{\mathrm{Al}}$.

\section{AlGaN superlattice}

The structure was grown by metal-organic chemical vapor deposition on the sapphire substrate. The intentionally undoped $\mathrm{Al}_{x} \mathrm{Ga}_{1-x} \mathrm{~N}$ layers with modulated Al content have been grown on the thick conductive layers (AlGaN:Si layer in sample C582 and GaN:Si layer in sample C594). The Al content, $X_{\mathrm{Al}}$, varied between 0.05 and 0.16. Semitransparent Schottky contacts have been evaporated on the surface. The ohmic contacts have been alloyed to make connection to the conductive layers.

The structures of the samples have been analyzed by X-ray spectroscopy. The measurements revealed the presence of additional peaks caused by superlattice. For example, one of the samples presented here (C582) has two layers containing superlattices with periods $\lambda_{\mathrm{SL}}=21 \mathrm{~nm}$ and $32 \mathrm{~nm}$ (see Fig. 1). The second sample described in this paper (C594) has a period $\lambda_{\mathrm{SL}}=33 \mathrm{~nm}$.

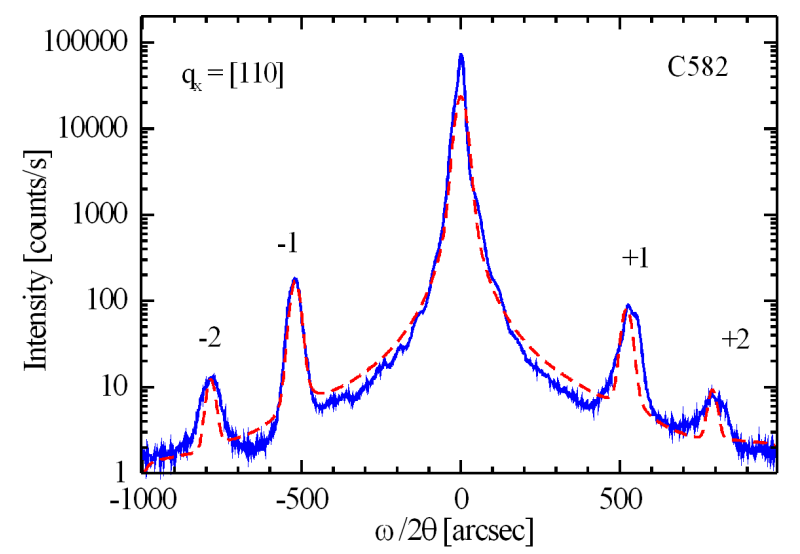

Fig. 1. X-ray diffraction curve of a sample containing the AlGaN SL. Solid line - experiment, dashed line - numerical simulation $\left(\lambda_{\mathrm{SL} 1}=32 \mathrm{~nm}, \lambda_{\mathrm{SL} 2}=21 \mathrm{~nm}\right)$. Sidelines $(-2,-1,+1,+2)$ are created by electromagnetic wave interference on the SL.

The determination of $\mathrm{Al}$ content is more difficult. For example, electroreflectance measurements of sample (C594) show three features corresponding to density of states maxima at $3.553 \mathrm{eV}, 3.588 \mathrm{eV}$, and $3.636 \mathrm{eV}$. Basing on these results, one can determine that $X_{\mathrm{Al}}$ oscillates between 0.05 and 0.08 (AlGaN parameters from [5]). On the other side, photocurrent spectra show broad bands corresponding to the $X_{\mathrm{Al}}$ range between 0.05 and 0.16 . 
Spontaneous oscillations of the composition can be explained under a few assumptions:

(1) Aluminum content $X_{\mathrm{Al}}$ depends upon potential at the surface $\Phi: X_{\mathrm{Al}}=$ $X_{0}+\alpha \Phi$. This assumption can be justified by experimental observations that the incorporation rate of many elements depends upon Fermi potential [6]. So, if the potential oscillates, Al content will also oscillate (see Fig. 2).

(2) It is necessary to take into account that electric field caused by spontaneous polarization is screened in the majority of the sample volume. However, there exists a layer of thickness $w$ just under the surface where electric field cannot be screened (see Fig. 2). In this case, the electric field, $E_{\mathrm{el}}$, at the point $z^{\prime}$ below the surface is equal

$$
E_{\mathrm{el}}\left(z^{\prime}\right)=\frac{P\left(z^{\prime}\right)-P(z+w)}{\varepsilon \varepsilon_{0}},
$$

where $P\left(z^{\prime}\right)$ and $P(z+w)$ are spontaneous polarizations at the point $z^{\prime}$ and at the distance $w$ below the surface. Spontaneous polarization is proportional to aluminum content $P=p X_{\mathrm{Al}}[1]$.

(3) Potential at the surface is an integral of $E_{\mathrm{el}}\left(z^{\prime}\right)$, so its oscillations are "delayed" in respect to $X_{\mathrm{Al}}$ and $E_{\mathrm{el}}$ (see Fig. 2).

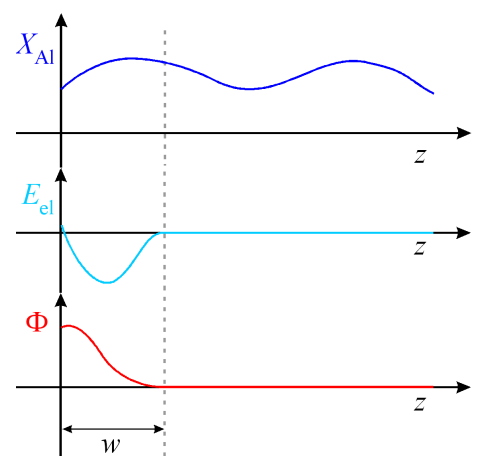

Fig. 2. Profiles of $\mathrm{Al}$ content $\left(X_{\mathrm{Al}}\right)$, electric field $\left(E_{\mathrm{el}}\right)$, and field-induced potential $(\Phi)$ during growth. Due to spontaneous polarization, $E_{\mathrm{el}}$ depends on $X_{\mathrm{Al}}$. Potential $\Phi(z)$ is an integral of $E_{\mathrm{el}}(z)$.

Taking into account the points (1), (2), and (3), one gets

$$
X_{\mathrm{Al}}(z)-\frac{p \alpha w}{\varepsilon \varepsilon_{0}} X_{\mathrm{Al}}(z+w)+X_{0}=\frac{-p \alpha}{\varepsilon \varepsilon_{0}} \int_{z}^{z+w} X\left(z^{\prime}\right) \mathrm{d} z^{\prime} .
$$

The solution of Eq. (2) is a periodic function $X_{\mathrm{Al}}(z)=X_{0}+A \sin \left(\kappa\left(z-z_{0}\right)\right)$. The oscillation period $\lambda_{\mathrm{SL}}=2 \pi / \kappa$ is proportional to $w$ and it is expected to be of the order of tens of nanometers. 


\section{Optical properties}

Photoluminescence (PL) measurements of the AlGaN superlattice show a comb of peaks, most probably related to the SL. These peaks are nearly temperature-independent which is in opposition to most PL peaks that decrease in intensity and shift to lower energy with increasing temperature. The light wavelength (inside GaN, $n=2.5$ ) of observed SL-related peaks is four times longer than the period of the SL (PL of shorter wavelengths cannot be emitted due to too low energy gap). The distance between the SL-related peaks in $k$-space is comparable with reciprocal of the SL thickness, so it resembles interference features. However, the peaks are significantly sharper than the expected interference pattern. Their shape is rather similar to multimode laser spectrum [7], which suggests that light passing through the superlattice is amplified. In fact, we observed a threshold in PL intensity vs. power dependence. The threshold power was rather low, below $1 \mathrm{~mW}$.

Photocurrent (PC) spectroscopy and Kelvin force microscopy (KFM) have been used in order to determine charge, field, and potential distributions in the structures. The KFM combines the idea of tapping mode atomic force microscopy (AFM) with a Kelvin probe to measure the distribution of the surface potential for conducting samples. Topography and potential are measured simultaneously "line by line" which enables us to correlate the potential and topography.

The observed PC spectra show two bands (see Fig. 3A). The low-energy band is narrow and corresponds to energy gap of material with minimal Al content $(3.53 \mathrm{eV})$. The high-energy band is broad and its energy corresponds to the whole range of $X_{\mathrm{Al}}\left(h \nu=3.6-3.9 \mathrm{eV}, X_{\mathrm{Al}}=0.07-0.16\right)$. At zero voltage the bands have opposite signs of photocurrent. At positive bias the high-energy band is practically absent. At negative bias, it increases exponentially (from about 1 pA to 100 pA when voltage $U_{\mathrm{B}}$ changes from 0 to $-8 \mathrm{~V}$ ). The effect is probably due to tunneling of photo-excited carriers through the barriers of superlattice.

Also the KFM spectra show that a sign of potential generated by the two bands varies with photon energy. The KFM technique gives also spatial resolution, so one can observe that the spectra depend also upon the position on the sample (see Fig. 3B). For example, inside the superlattice, high-energy band shows a negative sign and at the interface with the conductive layer both bands generate a positive potential.

Numerical calculations of potential, electric field, and charge distributions have been made for this structure. The potential and charge distributions can be calculated by self-consistent procedure taking into account that composition-dependent potential $\Phi_{X}$ adds to potential generated by electric charges (electrons $n$, holes $p$, and ionized defects $\left.n_{\mathrm{D}^{+}}, n_{\mathrm{A}^{-}}\right)$and spontaneous polarization $P_{X}[8]$ :

$$
\Phi(z)=\Phi_{X}-\frac{1}{\varepsilon \varepsilon_{0}} \int\left(P_{X}+e \int\left(-n+p+n_{\mathrm{D}^{+}}-n_{\mathrm{A}^{-}}\right) \mathrm{d} z\right) \mathrm{d} z .
$$




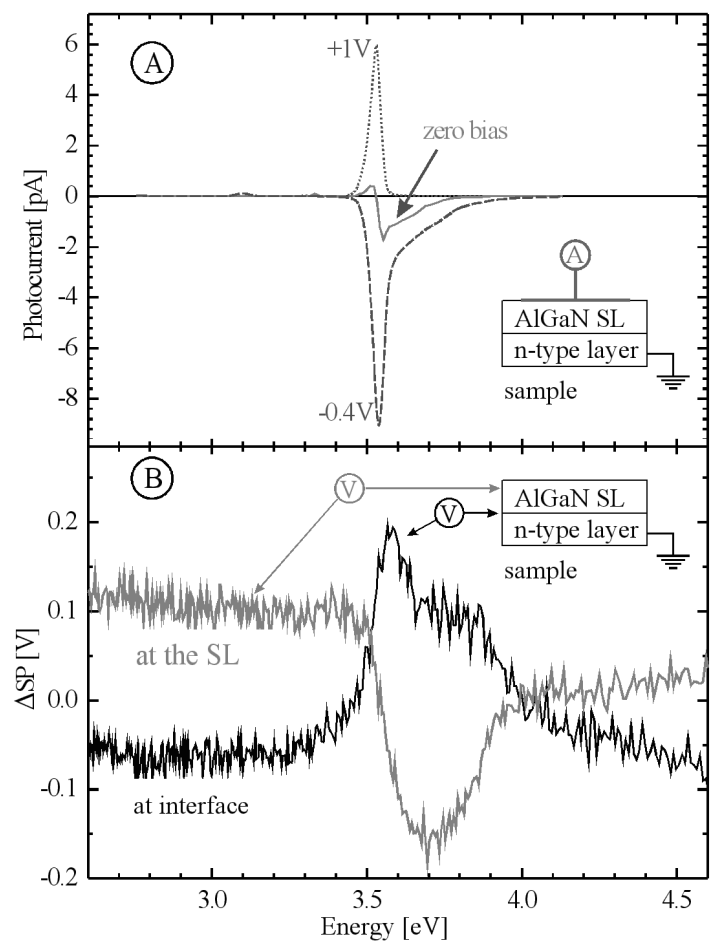

Fig. 3. (A) PC spectra measured at different biases. Zero-bias spectrum (solid line) shows that photocurrent is positive or negative, depending on photon energy. (B) KFM spectra measured at two different places.

Free carriers generated by light drive the system out of equilibrium. The excess concentration of carriers leads to their diffusion and creates photocurrent. In order to calculate carrier distribution and photocurrent, one has to solve Eq. (3) simultaneously with equations describing electron and hole transport:

$$
\frac{\partial n}{\partial t}=\mu_{\mathrm{e}} \frac{\partial n E}{\partial x}+D_{\mathrm{e}} \frac{\partial^{2} n}{\partial x^{2}}+G-R,
$$

where $\mu_{\mathrm{e}}, D_{\mathrm{e}}$ - electron mobility and diffusion constant, $G$ and $R$ - generation and recombination coefficients. A similar equation must be written and solved for transport of holes.

A computer model using these equations shows that electric field inside the structure (generated by spontaneous polarization and piezoelectric effect) cannot be efficiently screened by electrons (at room temperature) and oscillates with the phase opposite to the $\mathrm{Al}$ content. The field generates potential that adds to conduction band and valence band. The resulting potential oscillates with the same period but its phase is shifted. Consequently, the conduction band potential minima are shifted in respect to the valence band maxima and are in different 
positions than minima of $X_{\mathrm{Al}}$. The shift is about $8 \mathrm{~nm}$, which is about $25 \%$ of the SL period. Due to this effect, photo-generated holes and electrons are separated and localized in the areas of strong electric fields with different signs (see Fig. 4). Due to this separation, the product of electron and hole concentrations $\langle p n\rangle$ is about 5 times reduced (at room temperature), so the recombination rate is smaller which leads to longer lifetimes of photo-excited carriers. In this situation inversion of occupation and non-equilibrium effects can be achieved even at low excitation power density. The calculations have been made for room temperature. At growth temperature (about $1000^{\circ} \mathrm{C}$ ) the carrier concentration and diffusion are higher, so the field can be screened. On the other hand, at helium temperature localization of carriers is stronger and the effect of electron-hole separation is more dramatic than at room temperature.

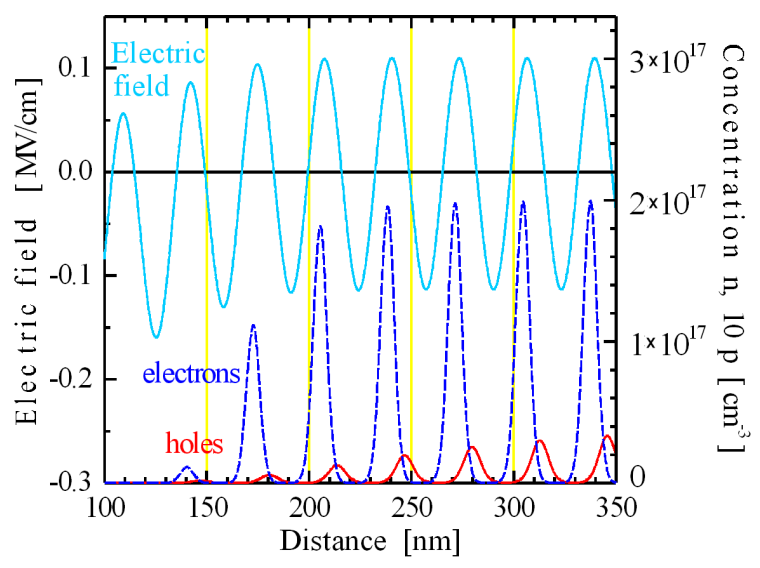

Fig. 4. Electric field (upper solid line, left axis) and charge concentration distributions (right axis) in the AlGaN superlattice. It is visible that holes (lower solid line) and electrons (dashed line) are separated.

Due to spatial oscillations of electric field, the photocurrent can have both directions depending upon depth of light penetration into SL. This effect explains strange current and potential spectra observed by photocurrent and KFM measurements.

\section{Conclusions}

Photocurrent spectra of the AlGaN superlattice show that light can generate currents of both directions positive and negative, depending on photon energy. The effect can be explained by spatial oscillations of electric field that changes direction coherently with the oscillations of $\mathrm{Al}$ content.

Due to the strong electric field, electrons and holes are separated and localized in the areas of strong electric fields with different signs. Separation of 
electrons and holes can lead to the inversion of occupation and non-equilibrium effects even at low excitation power densities. Sharp peaks present in photoluminescence spectra seem to confirm this supposition.

\section{Acknowledgments}

This work was partially supported by the State Committee for Scientific Research (Poland) grant No. 3 T08A 06328.

\section{References}

[1] F. Bernardini, V. Fiorentini, D. Vanderbilt, Phys. Rev. B 56, R10024 (1997).

[2] A. Drabińska, K.P. Korona, R. Bożek, J.M. Baranowski, K. Pakuła, T. Tomaszewicz, Phys. Status Solidi C 0, 329 (2003).

[3] D. Jena, S. Heikman, J.S. Speck, A. Gossard, U.K. Mishra, A. Link, O. Ambacher, Phys. Rev. B 67, 153306 (2003).

[4] K. Pakula, R. Bożek, J.M. Baranowski, J. Jasinski, Phys. Status Solidi C 2, 1073 (2005).

[5] E.V. Kalashnikov, V.I. Nikolaev, MRS Internet J. Nitride Semicond. Res. 2, a.3 (1997).

[6] G.F. Neumark, Phys. Rev. Lett. 62, 1800 (1989).

[7] M.P. Mack, A. Abare, M. Aizcorbe, P. Kozodoy, S. Keller, U.K. Mishra, L. Coldren, S. DenBaars, MRS Internet J. Nitride Semicond. Res. 2, a.41 (1997).

[8] K.P. Korona, A. Drabińska, A. Trajnerowicz, R. Bożek, K. Pakuła, J.M. Baranowski, Acta Phys. Pol. A 103, 675 (2003). 